\title{
III. Zur intrauterinen Therapie
}

\author{
von
}

\section{P. BRÖSE.}

In Heft 4 des IV. Bandes dieser Monatsschrift teilt $\mathrm{H}$ ofmeier einen Fall mit, in welchem nach einer intrauterinen kinspritzung von 50-prozentiger Chlorzinklösung mittelsı der 13 ra u n'schen Spritze der 'Tod cintrat und zwar wie Hofmeier annimmt, in Folge beginnender Peritonitis. Dass diese Frall an beginnender Peritonitis gestorben ist, scheint mir durchaus nicht sicher zu sein. Denn schwerlich wird sich bei einer Person, die am 24. Mär'z starb, beerdigt, nach 12 'Tagen wieder' ausgegraben und am 13. April secirt wurde, also am 20. Tage nach dem 'Torte, noch eine beginnende Peritonitis durch die Autopsie diagnosticieren lassen. Aber auch der klinische Verlauf, von welchem $\mathrm{H}$ ofmeier sagt, "dass nach demselben Niemand bezweifeln konne, dass in Folge des Einspritzens einer 50-prozentigen Chlorzinklösung in die Uterushöhle eine acute Peritonitis ermeugt worden ist", scheint mir durchaus nicht so absolut sicher für eine beginnende Peritonitis zu sprechen. Fs traten nach der Chlorzinkinjection „lieftige Schmerzen, ferner Frbrechen, Anschwellung des Leibes, Verfärbung des Gesichtes, Verschwinden des Pulses", also tiefer Collaps ein, alles Erscheinungen, die auch durch die blosse intrauterine Injection des (hlorzinks hervorgerufen sein konnten.

H of meier selbst wirft ja auch die Frage auf, ob es sich nicht "ausser der ortlichen Atzwirkung um eine direkt toxische Wirkung aut den Organismus" gehandelt haben kann. Bokelmann teilt mir einen Fall mit, in welchem fünf'Tage nach einer Uterusauskratzung nach einer mit grösster Vorsicht vorgenommenen Injection von 50-pro\%entiger Chlorzinklosung mittelst der Braun'schen Spritze in den Uterus unmittelbar darauf Collaps, Ohnmacht, Aussetzen oder Verschwinden des Pulses eintrat. Die Collapserscheinungen dauerten melirere Stunden; dann erst erholte sich die Frau. Auch ich habe ron diesem Fall den Eindruck einer toxischen Wirkung auf den Organismus, ähnlich dem Eiffekt, den eine Carbolinjection in das Rectum hervorruft. Dass unter Umstiinden eine onergische Resorption von der Uterushöhle aus stattfindet, beweisst ja auch die Hofmeier'sche Mitteilung über die Einwirkung des Alkohols seiner in den Uterus injicierten alkoholischen 10 -prozentigen Carbollösungen auf das Allgemeinbefinden der Frauen. Vielleicht wird man bei diesen Injectionen noch einmal die Einwirkung der starken Carbollösung auf den Organismus beobachten.

Welches nun die direkte 'Touesursache in jenem ron Hofmeier veröffentlichten falle gewesen ist, das ist nicht aufgekliirt; dass aber die Frau in Folge der intrauterinen Chlor/inkinjection \%u Grunde gegangen ist, daran wird Niemand zweifeln. So möchte anch ich mich der Warnung Hofmeier's ror der intrauterinen Einspritzung des Chlorzinks mittelst der Braun'schen sprit\%e in die Uterushöhle vollkommen anschliessen. 
Hofmeier kommt am Ende seiner Arbeit zu dem Schluss: "Fis ist also nicht die Anwendung der Braun'schen Spritze und die Einführung flüssiger Medikamente in grösserer Menge an sich, welche die Gefahr bedingen, sondern ungeeignete Anwendung ungeeigneter Medikamente." Ich will gern \%ugeben, dass die Anwendung der starken Chlorzinklösungen mittelst der Braun'scheu Sprit\%e eine ungeeignete ist. Ich habe das Chlorzink niemals so angewendet, und als ich die intrauterine Chlorzinkätzung mittelst der Play fa ir'schen Sonde, wie R h e in stadt er sie zuerst angegeben, empfahl ${ }^{1}$ ), auch gar nicht daran gedacht. dass derartige starke I.osungen in die I terushöhle eingespritzt werden würden. Deshalb aber auf ein so vorzligliches Medikament, wie es die starke Chlorzinklösung ist, in der gynäcologischen Therapie verzichten zu wollen, heisst das Kind mlt dem Bade ausschütten.

Die Anwendung der Bra un'schen Spritze und die Einführung flussiger Medikamente in grosserer Menge in den Uterus kann ich nicht für so harmlos halten, wie Hofmeier, und ich befinde mich darin in Ubereinstimmung mit anderen Gynakologen ${ }^{2}$ ), welche die Braun'sche Spritze ganz aus ihrem Armamentarium verbannt haben. Ich habe sowohl noch als Assistent von Schröder, wie auch später nach den Einspritzungen der doch fur ziemlich harmlos geltenden Jodtinctur trot\% vorhergehender Dilatation mittelst Schröder'scher Kupfersonden und vorhergehender desinfizierender Ausspïlung sehr unangenehme perimetritische Reizungen, ja grössere Exulate auftreten sehen. Die Möglichkelt des Übertritts von eingespritzter Fliissigkeit in die Bauchhöhle ist auch nicht so von der Hand zu weisen, wie es Hofmeier thut. Teilt doch Landa $\|^{1}$ ) einen Fall mit, in welchem Injectionen mit Bleiessig gemacht wurden und in der Peritonealhöhle der bald darauf Verstorbenen Schwefelblei nachgewiesen wurde. Ich benutze leshall, zur Nachbehandlung der Endometritis fungosa nach der Uterusauskratzung die Elektrolyse, die cin ausgezeichnetes Atzmittel und dabei ungefaihrlich ist.

I)as Chlorzink ist aber ein ausgezeichnetes Mittel für die katarrhalische Endometritis. Seit dem Jahre 1886 habe ich die intrauterine Chlorzinkätzung mittelst der mit Watte umwickelten Playfair'schen Sonde in vielen Hunderten ron liälen mit dem besten Erfolge angewendet. Der Einwand Hof ineier's, dass man dieses Aet\%mittel mittelst der Play fair'schen Sonde nicht in die Uterushöle bringen könne, weil bei dieser Inwendungsweise das Aetzmittel im Cervix abgestreift wiurde und nicht vber den innern Muttermund hinausdränge, ist rein theoretischer Art Die Praxis spricht dagegen. I as kann man fast mit der Sicherheit eines Experimentes nachweisen. Wenn man bei einer Frau nur den Cervix auf jene Weise atzt, so hat sie in den meisten Fällen crar keine Schmer\%en. I)ringt man aber in die Uterushöhle sin, su bekommern

1) Deutsche medicinische Wochenschrift $1 \times 88$.

2) Verhandlungen der Berliner medicinischen Gesellschaft 1894. Sit\%unir von, 8. November.

1) Verhandlungen der Berliner medicinischen Giesellschaft. 1. c. 
sehr viele Frauen starke Uteruskoliken, die stundenlang anhalten können, wenn sie auch nicht so stark sind, wie die Uteruskoliken, die nach Anwendung der Braun'schen Spritze entstehen. J. Veit ${ }^{1}$ ) führt ferner als Beweis dafur, dass man mit der Playfair'schen sonde Chlormink in den Uterus bringen kann, Folgendes an, woron sich Jeder leicht aberzengen kann. Wenn man eine mit chlorzink getrănkte Sonde nach der Winfiuhrung in den Uterus mit einer kleinen Verletzung am Finger ilırer Watte beraubt, so fiihlt man in der Wunde des Finger's das brennende Aet\%mittel deutlich genug.

Allerdings ein ganz gleichgiiltiger Eingriff ist die intranterine Aetzung mit einer so starken Chlor\%inklïsung - stark mïssen die lijsungen sein, sonst duchdringen sie das rïhe sekret nicht anch nicht. Anch hierbei muss man mit ier nötigen Vorsicht rerfahren. Nan muss natürlich Fälle mit Erkrankungen der Uterusanhänge von der Behandlung ausschliessen, man muss sich vor der Aetzung die Richtung des Uteruskanals genau mit der sonde bestimmen und darf endlich die Aetzumgen nicht zu häutig hintereinander vornehmen. Es geniigt vollkommen. wenn man sie alle \& 'lage ausfiilhrt; denn dann erst findet man den schorf der letzten Aetzung abgestossen. Ausserdem passiert es manchmal, dass sich ler Aetzschorf unter einer geringen, in seltenen Fällen sogar ziemlich starken Blutmng abstösst, die aber immer durch die Tramponade zil stillen ist.

Eine jede intranterine Therapie hat ihre Gefahren mol muss mit der nötigen Vorsicht angewendet werden. Anf keinen Fall ist diese Applikation des Chloryinks mittels der Play fair'schen sonde etwa mit grïsseren Gefahren verbunden, als die Apulikation anderer sogen. "geeigneter Flüssigkeiten" mittelst der Bram schen spritze. Auf einen Punkt mïchte ich aber bei der intrauterinen Behandlung der Endometritis catarrhalis hinweisen, der mir sehr wichtig $/ 1$ sein scheint: man soll iiberhaupt nicht in jerlem Fall von Fluor albus die ganze Uterushöhle in Angriff nehmen. Wenn man beim Cteruskatarrh in Speculum die Portio ansieht. so kamn man nicht ohne weiteres erkennen, wie weit der katarh in den Uterus hinaufgeht. ob der Katarh nur bis zum imnern Muttermund reicht oder anch die Uterushïhle ergriffen hat. In der Regel aher - das hat schon schröder golehrt und das habe ich auch aus meinen therapentischen lirfolgen gesehen - geht der Katarlh gramicht ïber den imnern Mutermund hinaus, und man sollte sich bei dem Fluor albus, besonders wenn er anf Gonnrophe berulit, in der Regel darauf beschränken, nu den Cervix zu behandeln. Fist, Wenn ich nach einigen Wochen sehe, dass ich so nicht \%um Ziele komme und auch damn erst nach Einlegung der sichultzeschen Probetampons mol Intersuchmog des Sekrets entschliesse. ich mich, die gan\%e Uterushöhle \% itten.

Hätte jener Arzt. der in dem ron $\mathrm{Hofmeier}$ veriffentlichten Falle wegen Fluor albus die jo-procentige Ghlor\%inklösung in die Vterushöhle injicierte, nur den cervix behandelt. so wiare er wahr-

1) Verh. der Berl. med. (iesellseh. 1894. 1. c. 
scheinlich vollkommen zmm Ziele gekommen, olıne dass ihm ein so tranuiges Ereignis passierte.

So kamn ich mich dem Ausspruch Hofmeiers, dass die "ungeeignete Anwendung ungeeigneter Madikamente" in diesem Falle die Gefahr bedingte, nicht gan\% anschliessen. Wohl war die Anwendungsweise und vielleicht anch die Indikationsstellung \%u verwerfen. Das Medikament selbst aher, das ich fiir ein sehr niitzliches halte, deshalb, weil es in ungeeigneter Weise angewendet wurde, als ungeeignet zu hezeichnen, das geht \%u weit. Auch das Morphimm ist ein Medikament, das in ungeeigneter Weise angewendet, den grössten Schaden stiften kamm. Und demnoch möchte wohl kein tr\%t auf das Morphium ver\%ichten.

\section{Zur intrauterinen Therapie.}

Erwiderung auf den Artikel ron $\mathbf{M}$. $\mathrm{H}$ of $\mathrm{meier}$ in der Monatsschrift für Goburtshülfe und Gynäkologie, Bd. IV, Heft 4.

Von

\section{A. DÜHRSSLN.}

Ich stimme mit Herrn Hofmeier vollstăndig uberein, wenn er am Schlusse seines Aufsat\%es Folgendes sagt: "Aus dem (iesagten geht hervor, dass man, um ïberhaupt eine Wirkung auf die Gewebe zu er\%ielen, jedenfalls konzentrierte liosungen anwenden muss und auch unter Beobachtung der nötigen Vorsichtsmassiegeln olıne Schaden allwenden kann"; und weiter: "Ls ist also nicht die Anwendung der Braun'schen Spritze und die Einfïhrung Hiissi Medikamente in grösserer Menge an sich: welche die Gefahr bedingen, sondern ungreeignete Anwendung ungeeigneter Medikamente "

I)iesen Anschaumgen glaube ich durch die in meinem gyna. kologischen "Vademecum" niedergelegten Vorschriften rollikommen Rechnung getragen zu haben und begreife daher nicht, we Hof meier mir eine gewisse Verantwortlichkeit fur den ron ihm berichteten 'Todesfall nach 50pro\%entiger Chlor\%inkinjektion aufbiirden will. Hofmeier verlangt, „dass dicjenigen Autoren, welche derartige heroische Mittel, wie Injektionen ron 50pro\%entiger Chlorzinklossung, literarisch empfehlen, ein wenig mehr die ausserordentliche Verantwortlichkeit, welche sie dadurch unter L'mständen auf sich laden, erwägen und zum Mindesten die notwendigen oder uncrlässlichen Vorsichtsmassregeln in einer unverkennbaren und bestimmten Weise gleich dabei \%um Ausdruck bringen."

Diese Vorsichtsmassregeln sind von mir in ler ron $\mathrm{H}$ of meier ritierten Stelle (Gynäkologisches Vademecum, 4. Aufage, S. 101 u. 102) in unverkennbarer und bestimmter Weise zum Ausdruck grebracht, indem ich vor und nach der Injektion eines A etzmittels - ich nenne S. 101 Jodtinktur, Iiq. ferri, Chlorzink 